\title{
Inhaltsverzeichnis Jahrgang 1918
}

\section{Abhandlungen und Notizen.}

Bondi, J. E.: Zu Sachau APO 4, 10 . . . . Bork, F.: Zum Jahresrätsel der Achiqargeschich te Christian, $\nabla .:$ Neuarabisch iğr "Fuss" Dombart, Th.: Der Turmbau zu Babel Erbt, W.: Die Chronologie des ersten nachexilischen Jahrhunderts

- Zu F. E. Peisers Jesaja Kap. 9“ ${ }^{\circ} \cdot \dot{D}^{\circ}$

- Eine Mond- und Sonnen-Finsternis im $\mathrm{AT}$

Geller, S.: Das babylonische Neolitikum . .

Hüsing, G.: Kaspisches IV . . . . . . . .

- Zeichen $-E Y=$ hir. . . . . . . . .

- Kaspisches $\nabla$

Eumbert, P.: Der Deltafürst So'

Jirku, A.: "Hebräische" u. ,israelitisch $\theta^{\alpha}$ Sk Skaven - Der assyrische Name des Benhadad III .

Kletzel, W.: אב im Munde von Frauen . .

Landersdorfer, S.: Zur Etymologie von hel

Lohmann-Eaupt, C. F.: Zur Ermordung Sanheribs.

Madeja, E. Das Ninlil-Tor zu Ninive.

Marstrander. C.: VAT $7478 \mathrm{Kol}$. III $30 \mathrm{ff}$.

Meissner, B.: Eine babylonische Stele Assurbanjpals (?) . . . . . . . . . . . . .

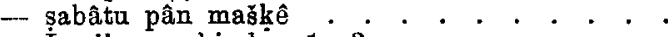

- Lexikographisches $1-3$

- Umma.

- Šamašsibni von Bît-Dâkûri

- Lexikographisches 4

Peiser, F. E.: Jaudi

Perles, F : Ergänzungen zu den Akkadischen Fremdwörtern"

- Zur Aussprache von "הוה"

" Zor Aus Schmidkte, Fr.: Dor Ort d. Errmordung Sanheribs Schollmeyer, A.: Zur Serie harra = hubullu .

Schroeder, O.: Ein mündlich zu bestellender altbabylonischer Brief

- Za den Königslisten von Assur.

- Chronologische Miszellen

- Veber die Glossen ji-ir (ma) und mar-ia-nu(ma)

- Zur Götterliste für den Schulgebrauch . . .

- Reste der Sprache von Hanigalbat . . . .

- Ueber die ältesten Münzen . . . . . .

Schultz, Wolfg.: Iranisches bei Berossos . 227.

Ungnad, A.: Der Gottesbrief als Form assyr. Kriegsberichterstattung

- Ein merkw ürdiges sumerisches Liebnwort.

- Sumerische Handerhebungsgebete . . . . .

- Nabu und Nimurta.

- Zur fünften Tafel dor Serie HAR-RA

Wiedemann, A.: Trinken durch einen Schlauch

\begin{tabular}{r|} 
Spalte \\
17 \\
226 \\
128 \\
161 \\
6.33 \\
78 \\
176 \\
209 \\
43 \\
76 \\
264 \\
224 \\
81 \\
279 \\
1 \\
175 \\
\\
273 \\
165 \\
128 \\
119 \\
124 \\
171 \\
219 \\
220 \\
272 \\
48 \\
65 \\
129 \\
130 \\
169 \\
174 \\
5 \\
41 \\
75 \\
125 \\
127 \\
274 \\
257 \\
72 \\
115 \\
116 \\
167 \\
224 \\
280 \\
\end{tabular}

Besprechungen.

Spalto

Aurich, Gust.: Hagios Nikolaos (C. Fries) . 245

Bang, W.: Zu den türkischen Zeitbestimmungen (Heinr. Winkler) . . . . . . . . . . 286

Baumgartner, W.: Die Klagelieder des Jeramia

(M. Lö hr) (Hans Rust) . . . . . . . . .

Cassel, D.: Hebräisch-deutsches Wörterbuch (M. Löhr) Gottes (S. Landersdorfer) . . . . . Eichrodt, W.: Die Quellen der Genesis (M. Lö hr Eissfeldt, $O .:$ Erstlinge and Zehnten in AT (M. Löhr)

Emin Pascha siehe Stuhlmann

Figulla, H. H. a. F. F. Weidner: Keilschriftterte aus Boghazköi 1. u. 2. H. (B. Meissner)

Friedmann, M.: Sifra (S. Poznanski) . . Geller, S.: Die sumerisch-assyrische Serie Logal-e (0. Schroeder) · - C D :

Glück, H.: Die beiden "sasanidischen Drachen" (0. Schroeder) . . . . . . . . . .

Gressmann, E.: Das Weibnachtserangelium (W. Erbt) $\cdot \cdot \cdot \cdot \cdot \cdot \cdot \cdot \cdot \cdot$ Grothe, W.: Türkigch-Asien u. s. Wirtschaftswerte (R. Bartmann) (A. Wiedemann) . . . . . . . Hasenclever, Ad.: Geschichte Aegyptens in XIX. Jahrh. (M. Sobernheim) Hell, Jos.: Die Religion des Islam (H. Rust)
Hoernes, M.: Urgeschichte der bildenden Kunst (Moernes, Mancritius)
(M. Panchte der bildenden Kunst Eopkins, W.: Epic Mythology (I.Scheftel ow itz) Eorovitz, S.: Siphre (S. Poznański). . . Horten, M.: Einführung in die türkische Sprache and Schrift (Fr. Schwally). . . . . .

Hussey, Mary Inda: Sumerian tablets in the Harvard Semitic Museum (W. Förtsch) . . 180

Imhoff: Die türkische Heeresmacht (C.F.Seybold) 149

King, L.: A history of Babylon (B. Meissner) 86

Klein, Otto: Syrisch-griechisches Wörterbuch zu den vier kanonischen Evangelien (Br.Violet)

Krause, P. R.: Die Türkei (Fr. Schwally) .

Kubitschek, Wilhelm: Zur Geschichte von Städten d. römischen Kaiserreichs (Arth. Mentz)

Langer, Fritz: Intellektual-Mythologie (Carl Fries) . . . . . . . . . . . 297

$\begin{array}{cr}\text { Fries) } \\ \text { Merz, \#.: D. Blutrache b. d.Israeliten(W.Caspari) } & 83\end{array}$

Messel, N.: Die Einbeitlichkeit der jüdischen Eschatologie (B. Violet)

Mitteilungen des Seminars für Orient. Sprachen
zu Berlin, 19. Jabrg. 2. Abt. (R. Hartmann) zu Berlin, 19. Jabrg. 2. Abt. (R. Hartmann)
20. Jahrg. 2. Abt. (R. Hartmann) . . . .
145 290

86

141

86
49

18

185

$2: 3$

134

99

91

246

243

104

99

51

29

פ34

57

$-287$

143

145

191 
Moritz, B.: Der Sinaikult in beidnischer Zeit (E. Brandenburg) $\dot{2}$ Jahrg. (J. Herrmañ) Palästinajahrbuch, 12. Jahrg. (J. Herrmann)
Peez, C. $\nabla .:$ Ein türkischer Grossvezier aus Graz

(R. Hartmann) (W. Förtsch)

Schlögl, N. J.: Ijjob (I. L Löw) Schmidtke, F.: Asarhaddons Statthalterschaft in Babylonien (O. Schroeder)

Schuimann, L.: Zurtürkischen Agrarfrage $(\dot{M} \cdot \dot{L}$ ö $\dot{h} r)$ Söderblom, N.: Das Werden des Grottesglaubens (Marie Pancritius)

Strack, H. L.: Jüdisches Wörterbuch; Jüdjsché

Texte (F. Perle日) Stuhlmann, Franz: Tagebücher von Emin Pascha (E. Brandenburg). . . . .

Theis, Johannes: Die Weissagung des Abdias (J. $\mathrm{H} \theta \mathrm{hn}$ ) .

Thomeen, P.: Denkmäler Palästinas a. d. Żit

Jesu (A. Allgeier) . . . . . . . . . .

Timme, P.: Tell el-Amarna vor d. deutschen Ausgrabung (W. Wreszinski)

Unger, E.: Die Stele des Bel-Haran-beli-ussur

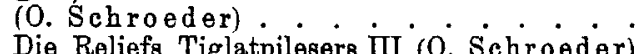

- Die Reliefa Tiglatpilesers II (O. Schroeder)

stellungen ( $W$. Wreszinski) . . . . . .

Wessely, C.: Duodecim prophetarum minorum vers. Achmimicae, cod. Rainerianus ed. C. W. (W. Spiegelberg). . . . . . . . .

\section{Verzeichnis der Rezensenten.}

Allgeier, A.

Brandenburg, E.

Caspari, $W$

Erbt, W.

Förtsch, W.

Fries, C. . . . . . . 245

Hartmann, R. . . . . . . 99. 145. 191. 244

Hehn, J. . . . . . . . . . . . 283

Herrmann, J. . . . 85

Landersdorfer, $\mathrm{s}$.

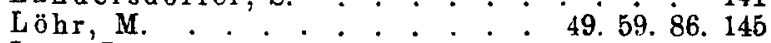

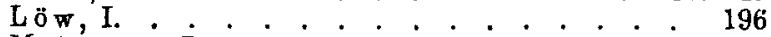

Meissner, B. . . . . . . . . . . 18.86

Mentz, Arthur . . . . . . . . . 287

Pancritius, M. . . . . . . . . . . . . . . . 104

Perles, F. . . . . . . . . . . . . 196

Poznañski, S. . . . . . . . . . . . . 50.51

Rust, H. . . . . . . . . . . . . . . 243

Scheftelowitz, I. . . . . . . . . . . . 99

Schroeder, O. . . . . . . . 48. 135. 185. 243

Schwally, Fr. . . . . . . . . . . . . 29.57

Seybold, C. F. . . . . . . . . . . . . 149

Sobernheim, M. . . . . . . . . . 246
Spiegelberg, Whalto

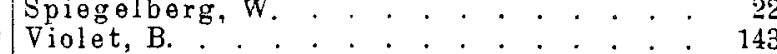

85 Wiedemann, A . . . . . . . . . 91

Winkler, Heinrich . . . . . . 286

Wreszinski, W.

236

Caspari, W. . . . . . . . . . 204

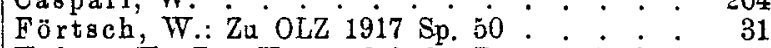

Holma, H.: Zum Verständnis des Papyrus Sachau 204

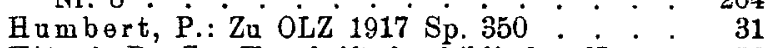

Kittel, R.: Zur Umschrift der biblisehen Namen 59

Meissner, Br.: Berichtigung zu OLZ $1918 \mathrm{Sp.} 299$

Peiser, F. E.: Zn Sp. 62 Anm. 1 . . . . . 106

- Zu OLZ Sp. 205 . . . . . . . . . . . . 249

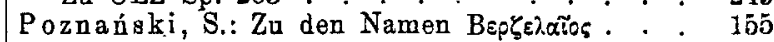

50 Aegypten 61. - Griechenland 61. - Italien 106. Russland 106.

\section{Aus gelehrten Gesellschaften.}

Vorderasiatische Gesellschaft 107. - Academie des Inscriptions et Belles-Lettres 107. 249. - Berliner Akademie der Wissenschaften 250.

\section{Mitteilungen.}

Fragmente der Res gestae Divi Augusti 61. - Urkundenbibliothek f. d. Geschichte des Islams 61. - Erwerbung d. K. Musen z. Berlin 61. - Seminar f. Orient. Sprachen 61. - Institut f. Islam und osteuropäische Geschichte 106. - Fondation de Goeje 155. - Vereinbarung zwischen R. Asiatic Soc. und Soc. Asiatique 155. - Archäologische Schule in $\mathrm{J}_{\theta-}$ rusalem 251 .

\section{Personalien.}

Bittner, Max 155. - Chavannes, Ed. 107. - Dalman, Gust. 61. - Faik-Bei 31. - Frech, Fr. 31. - Gelzer, M., 251. - Missis Grenfell 289. - Hartmann, R. 204. - Hess, J. J. 107. - Karabacek, Josef von 289. King, L. W. 107. - Klausner, J. 204. Kolbe, W. 251. - Kornemann 107. - Legrain, G. 61. Lehmann-Haupt, C. F. 251. - Lütke, L. 204. - Mann, O. 107. - Mez, Adam 61. - Radloff, W. 251. - Reisner, G. A. 107. - Schwab, Moïse 107. Steller, E. 204. - Thierry, G. J. 204. - Thiersch, Herm. 155. - Wellhausen, J. 61. - Winkler, Heinrich 251.

Zeitschriftenschau Am Schlusse jeder Nuwmer. 